\title{
MS44-P08 | Utilising MAXRd In THe Study of Inclusion Compounds
}

Ramsnes, Stian (University of Stavanger, Stavanger, NOR); Larsen, Helge Bøvik (University of Stavanger, Stavanger, NOR); Thorkildsen, Gunnar (University of Stavanger, Stavanger, NOR); Nicholson, David Graham (Norwegian University of Science and Technology, Trondheim, NOR)

The Mathematica X-ray diffraction package MaXrd has now been expanded with the capability to compose custom crystal structures, particularly aimed at facilitating the embedment of a guest phase into a host lattice. After importing the required crystallographic information from a cif file, one can extend the asymmetric unit to a desired number of unit cells while inserting atoms, molecules or other structures in the process. The embedded phase can also be distorted and/or rotated by a specified or random amount when placed into the host. The resulting structure can be visualised in three dimensions in direct space and the information may be utilised automatically by DISCUS to obtain a simulated diffraction pattern. A consequence of this technique is that the space group of the guest phase becomes independent of that of the host (essentially having $P 1$ symmetry). This gives the means to test hypotheses on the crystal structure and simultaneously investigate reciprocal space for any implied characteristics in a relatively swift and easy manner. This functionality is used in our ongoing study of a thioureaferrocene clathrate, which has proven challenging with regard to its phase transitions and the five-fold symmetry of the cyclopentadienyl rings. 